\title{
Sistemas de Indicadores de Sustentabilidade:uma aplicação do Ecological Footprint Method no município de Campina Grande (PB)
}

\author{
Indicators Systems of Sustainability: an application of Ecological Footprint \\ Method in Campina Grande (PB)
}

\author{
Maria José da Silva Feitosa' \\ Gesinaldo Ataíde Cândido² \\ Luciene Alencar Firmo ${ }^{3}$
}

\section{Resumo}

A problemática que envolve a sustentabilidade do planeta é fator preocupante para humanidade, uma vez que a capacidade do ambiente suportar impactos decorrentes das ações humanas está se esgotando. Assim, estudar a sustentabilidade - por meio das ferramentas de gestão - é relevante, na medida em que colabora para reduzir impactos sobre a natureza e promove o desenvolvimento sustentável. O objetivo deste artigo é analisar a sustentabilidade do município de Campina Grande, utilizando o Ecological Footprint Method. Este método mensura a pressão sobre os recursos naturais a partir de itens específicos de consumo. A pesquisa realizada é exploratória e descritiva orientada por estudo de caso, utilizando dados secundários referentes a indicadores relacionados ao consumo de energia elétrica, água, geração de resíduos e combustíveis. Os resultados apontaram que o metabolismo da cidade requer uma área 449,03 vezes maior que o seu território para suprir a sua demanda referente aos itens de consumo utilizados.

Palavras-chave: sustentabilidade; meio ambiente; Ecological Footprint Method.

\section{Abstract}

Problems involving the planet sustainability are a factor for humanity concern, since the ability to withstand environmental impacts resulting from human actions

I Administradora pela Universidade Federal de Campina Grande, UFCG; Pesquisadora do Grupo de Estudos em Gestão, Inovação e Tecnologia - GEGIT/UFCG; Rua Duque de Caxias, 539. Bairro: Prata; 58400-506, Campina Grande, Paraíba, Brasil; E-mail: mjsfeitosa@gmail.com

2 Dr.; Administrador; Professor Titular da Unidade Acadêmica de Administração e Contabilidade da Universidade Federal de Campina Grande, UFCG; Bolsista de Produtividade em Pesquisa do CNPq; Lider do Grupo de Estudos em Gestão, Inovação e Tecnologia - GEGIT/UFCG; E-mail: gesinaldo@pq.cnpq.br

3 Administradora pela Universidade Federal de Campina Grande, UFCG; Pesquisadora do Grupo de Estudos em Gestão, Inovação e Tecnologia - GEGIT/CFCG; E-mail: lu_catole@hotmail.com

Recebido para publicação em 30/08/2010 e aceito em 27/10/2010

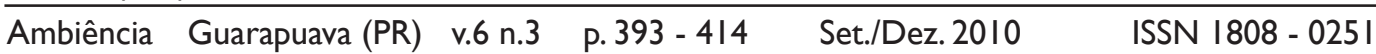


has been exhausting. Thus, studying the sustainability - through the management tools - is relevant in that it helps to reduce impacts on the nature and promote sustainable development. This article aims at examining the sustainability of the city Campina Grande, using the Ecological Footprint Method. This method measures the pressure on natural resources from specific items of consumption. The research conducted is focused on an exploratory and descriptive case study, using secondary data for indicators related to consumption of electricity, water, generation of waste and fuels. The results showed that the metabolism of the city requires an area 449.03 times larger than its land to meet their demands related to items of consumption.

Key words: sustainability; environment; Ecological Footprint Method.

\section{Introdução}

Visando à ascensão econômica e ao progresso, o homem desde os primórdios da humanidade executa atividades que afetam direta e significativamente o meio ambiente. Inicialmente, essas atividades eram realizadas de forma rústica e com pouca intensidade, em virtude da inexistência, na época, de um acervo tecnológico e informacional suficiente para executar grandes transformações na natureza. Com o passar dos anos, o ser humano desenvolveu técnicas e equipamentos que o auxiliaram na exploração dos aspectos naturais.

Seguindo o compasso dos avanços tecnológicos, a deterioração do meio ambiente começou a se agravar, de fato, com a Revolução Industrial do século XVIII e, principalmente, com a abertura, expansão e dinamização dos mercados mundiais em meados do século XX, tendo em vista que ocorreu a estruturação e o fortalecimento dos sistemas produtivos em virtude dos constantes avanços tecnológicos que ocorreram na época.

$\mathrm{O}$ aumento da demanda de produtos proporcionou o crescimento da margem de lucratividade das empresas, as quais passaram a focalizar o lucro como principal fonte de crescimento. Neste sentido, o lucro passa a ser visualizado como principal objetivo das empresas, em detrimento dos aspectos sociais e ambientais.

Paradoxalmente ao modelo de crescimento econômico vigente, eclodem os problemas ambientais e trazem em si um desafio sem precedentes para toda a humanidade: os ecossistemas da terra não têm capacidade para suportar os níveis de atividade econômica e de energias atuais, uma vez que a capacidade de carga do planeta está no limite (CINDIN; SILVA, 2004). Em outras palavras, a pressão da carga humana em relação à capacidade de suporte do ambiente natural é desproporcional, isto é, as fontes naturais não conseguem repor os recursos na mesma proporção que as atividades humanas vêm degradando.

$\mathrm{Na}$ medida em que se percebe que a capacidade de carga do planeta está se esgotando, começa a emergir uma forte preocupação, por parte da sociedade, no que se refere à sua própria sobrevivência, bem como das futuras gerações. As pessoas começam a questionar o padrão atual de consumo, sobretudo, a partir de 1970, quando começam a surgir os primeiros estudos e pesquisas sobre a sustentabilidade como forma de desenvolver alternativas que 
possam contribuir para redução dos impactos ambientais.

Assim, ao se observar o estágio de degradação no qual a natureza se encontra, as pessoas começaram a questionar até quando os recursos naturais serão capazes de suportar a pressão exercida pelo homem. Essa pressão é resultado do crescimento populacional, consumo inconsciente e da intensa geração de resíduos. Em meio às discussões e percepções acerca da questão ambiental, novas formas de consumo e de relacionamento com o ambiente estão sendo propostas pela própria sociedade, bem como por instituições de fomento na busca de reduzir significativamente a dependência humana dos recursos naturais.

Para auxiliar na obtenção de novas formas de relacionamento, estão sendo estudados mecanismos capazes de prevenir e controlar a degradação ambiental por intermédio de investimentos no desenvolvimento de novas tecnologias, como também, modelos de avaliação, por exemplo, os indicadores de sustentabilidade. Estes são utilizados para aferir os impactos provocados pela interferência humana no meio ambiente, os quais são perfeitamente corroborados nos ecossistemas urbanizados. Dentre uma série de ferramentas de mensuração utilizadas, o Ecological Footprint Method (Método da Pegada Ecológica), proposto por Wackernagel e Rees (1998), destaca-se pelo fato de ser uma ferramenta simples (no que se refere a pouca complexidade na sua utilização) utilizada para medir, comunicar e comparar o desenvolvimento das nações através da mensuração da sustentabilidade.

A referida ferramenta possui princípios que consistem em contabilizar os fluxos de matéria-prima e energia existentes em um determinado sistema, convertendo-os de for- ma adequada em áreas produtivas de terra ou de água (WACKERNAGEL; REES, 1996). Conforme Dias (2002, p. 182) o método da pegada ecológica "permite estabelecer de forma quantitativa um diagnóstico dos resultados das atividades humanas desenvolvidas junto ao socioecossistema e os custos em termos de apropriações de áreas naturais, para manutenção do terrametabolismo".

Neste contexto, o objetivo deste artigo é analisar a sustentabilidade do município de Campina Grande por intermédio do Ecological Footprint Method (Método da Pegada Ecológica), procurando obter os dados dos principais itens de consumo que compõem a maior parte da pressão exercida sobre os recursos naturais do Município objeto de estudo. Neste contexto, a sustentabilidade pode ser entendida como a capacidade de um sistema suprir as necessidades da geração presente sem impossibilitar as futuras gerações de suprirem as suas próprias necessidades (RELATÓRIO DE BRUNDTLAND, 1987).

Desse modo, a presente pesquisa pode ser classificada como descritiva e exploratória conduzida por meio de um estudo de caso, no qual foi aplicado o instrumento de mensuração denominado método da pegada ecológica. Para a coleta de dados foi utilizado um conjunto de dados secundários, os quais foram posteriormente contextualizados e analisados em conformidade com os parâmetros estabelecidos pelo método.

\section{Revisão de Literatura}

\section{O homem, a natureza e as cidades}

A interação do homem com a natureza no decorrer dos anos tem alterado negativamente o ecossistema natural, uma 
vez que as atividades executadas por esse indivíduo consomem grandes áreas produtivas do planeta, diminuindo a proporção per capita desses espaços ecológicos rurais e urbanos (SILVA; SANTOS, 2007).

Nos territórios urbanos, a problemática ambiental é perfeitamente visível, uma vez que o nível de consumo e consequente geração de resíduos são intensos, em virtude, sobretudo da grande quantidade de pessoas que vivem nestas áreas. Dessa forma, as zonas urbanas podem ser visualizadas como "pontos quentes", tendo em vista que um hectare de área metropolitana consome mais de 1000 vezes a energia de uma área semelhante utilizada em um ambiente natural (O'MEARA, 2000).

Além disso, as cidades são visualizadas como sistemas dependentes de outros sistemas porque, geralmente, produzem pouco ou nenhum alimento e outros materiais orgânicos, poluem o ar, as águas, e não reciclam os materiais inorgânicos que produzem. Em outras palavras, as áreas urbanas não são capazes de produzir o que a sociedade necessita, e muito menos assimilar todos os dejetos gerados pelas pessoas em decorrência de consumo.

Nessa perspectiva, Cindin e Silva (2004) esclarece que as cidades - resultantes da modernização da sociedade - contribuíram consideravelmente para que o relacionamento entre o homem e a natureza se fundamentasse em princípios de exploração, nos quais a natureza é apenas uma fonte de recursos utilizados para o crescimento econômico das nações. Dessa forma, o desequilíbrio nas relações entre meio ambiente e sociedade provoca sérios problemas ambientais.

Dessa maneira, são nas relações sociais e na possibilidade de transformação das mesmas, que repousam as alternativas para resolução deste impasse contemporâneo. $\mathrm{O}$ que se deseja é o planejamento e a organização do espaço, de modo a garantir a qualidade de vida de toda população. $\mathrm{O}$ surgimento de novas formas de relacionamento entre homem e natureza, bem como a o planejamento e a organização do espaço, são alternativas inteligentes decorrentes da iniciativa integrada formada por sociedade, instituições (públicas e privadas) e governos. Estes, por sua vez, começam a se preocupar com os problemas ambientais a partir do momento que grandes investimentos têm sido realizados com a finalidade de remediar algumas catástrofes naturais que têm ocorrido por todo mundo (MORAES, 2007)

Neste contexto, surge a necessidade de educar as gerações presentes e futuras de modo que venham a agir com responsabilidade e sensibilidade, conservando o ambiente saudável no presente e para o futuro (QUEIROZ et al., 2000). Muitas ações estão sendo desenvolvidas por órgãos de pesquisa, universidades e instituições não governamentais no sentido de deixar claro para a população mundial que preservar, nos dias atuais, é questão de sobrevivência. Além disso, esses órgãos se baseiam em uma diversidade de estudos e análises, que os auxiliam na proposição de alternativas para possíveis soluções.

Assim, mudanças de comportamento podem ocorrer na medida em que ações sejam introduzidas em todos os âmbitos da sociedade de forma sistêmica, com o auxílio de elementos tal qual a educação ambiental, de maneira a despertar na sociedade o interesse em preservar a natureza e a cobrar das instituições governamentais e privadas o desenvolvimento e implementação de novas formas de gestão capazes de envolver políticas públicas no sentido de 
reduzir significativamente a degradação ambiental (PENNA, 1999). É necessário que haja um equilíbrio nas relações entre homem e natureza de modo a promover um desenvolvimento que abarque as dimensões: ambiental, social, cultural, institucional, e econômica, pois o desenvolvimento é validado quando ocorre de forma integrada.

$\mathrm{Na}$ visão de Dias (2002), muitos objetivos, internacionais e nacionais direcionados ao meio ambiente, não serão atingidos sem uma reforma política extensiva e mudanças significativas nas práticas e estratégias atuais. Parte do desafio é, no entanto, criar mecanismos legais mais rígidos que garantam a fiscalização, bem como, a utilização de técnicas, a exemplo dos sistemas de indicadores, que permitam avaliar e acompanhar as mudanças ocorridas no ecossistema, contribuindo para o surgimento de um novo modelo de desenvolvimento denominado sustentável. Os sistemas de indicadores são formados por ferramentas utilizadas para mensuração da sustentabilidade de determinadas localidades. Tais ferramentas são denominadas indicadores de sustentabilidade.

\section{Indicadores de sustentabilidade}

Utilizados para refletir determinada situação de um sistema, através da mensuração do desempenho de aspectos que a envolvem, os indicadores são parâmetros selecionados e considerados isoladamente ou combinados entre si, sendo especialmente úteis para expressar as condições de um sistema em análise (GONÇALVES et al., 2000).

Para Mitchell (1997), um indicador é uma ferramenta que permite a obtenção de informações sobre uma dada realidade e tem como principal característica o poder de sintetizar um conjunto complexo de informações retendo apenas o significado essencial dos aspectos analisados.

$\mathrm{Na}$ avaliação da sustentabilidade, os indicadores são importantes, sobretudo, porque ajudam a relatar as condições de sistemas complexos e interdependentes, bem como promovem a avaliação de desempenho de diversas maneiras de gestão e políticas aplicadas em uma localidade visando alcançar a sustentabilidade. Dessa maneira, os indicadores são instrumentos eficientes para despertar nas pessoas a atenção para alterações nos sistemas sociais econômicos e ambientais (McCOOL; STANKEY, 2004).

Atualmente, a elaboração de instrumentos de mensuração é um dos principais desafios na construção de um desenvolvimento sustentável, na medida em que eles auxiliam a tomada de decisão nos níveis global, nacional, regional e local. Tais instrumentos, denominados indicadores de sustentabilidade, são ferramentas de mensuração e avaliação constituídas por uma ou mais variáveis que, associadas através de diversas formas, expressam significados mais abrangentes sobre fenômenos a que se referem. Um indicador deve ser entendido como um parâmetro, ou um conjunto de parâmetros que fornecem informações sobre um determinado fenômeno, com uma extensão significativa (IBGE, 2004).

Sendo assim, tais ferramentas são consideradas instrumentos essenciais para guiar a ação e subsidiar o acompanhamento e a avaliação do progresso alcançado rumo ao desenvolvimento sustentável. Esses indicadores têm sido enfatizados a nível internacional em virtude, sobretudo, da preocupação com a problemática social e ambiental, além de ter a vantagem de poder expressar a realidade através de gráficos, 
de modo a refletir tendências e determinar previsões futuras.

Em virtude da visão integrada de determinada realidade local, os indicadores de sustentabilidade são capazes de alertar sobre problemas antes que eles se tornem muito graves (BELLEN, 2007).

São muitas as ferramentas que podem ser usadas para mensurar o desenvolvimento sustentável, de modo que sua escolha depende do ambiente e contexto no qual será aplicado. No caso deste trabalho, a ferramenta usada foi o Ecological Footprint Method - EFM, levando em consideração que este é um instrumento capaz de refletir uma realidade local e de analisar os impactos causados pelas atividades humanas. Além disso, o referido método é uma ferramenta incluída dentro de uma categoria de sustentabilidade considerada forte, a qual está fundamentada na preservação ambiental e na qualidade de vida, propondo uma mudança radical na atitude da sociedade e no modelo econômico de consumo, de modo a respeitar os limites da natureza, ou sua "capacidade de carga" (BELLEN, 2007).

\section{Ecological Footprint Method - EFM}

A capacidade de carga do sistema mantém uma relação direta com a utilização excessiva e desmedida dos recursos naturais, na medida em que o consumismo exacerbado está promovendo a degradação ambiental e a produção de resíduos, caracterizando a falta de comprometimento da humanidade em relação à natureza (BELLEN, 2007).

Dessa maneira, os autores Wackernagel e Rees (1998) elaboraram o método Ecological Footprint Method (Método da Pegada Ecológica) em 1996 por ocasião do livro Our ecological footprint, almejando medir o impacto que os indivíduos de uma determinada população provocam sobre o sistema global para sustentar o seu estilo de vida.

Conforme Bellen (2007), o Método da Pegada Ecológica é um instrumento de contabilidade ambiental, que objetiva mostrar com maior clareza a relação entre uma unidade populacional e a natureza, a capacidade de renovação dos recursos, a capacidade de suportar impactos ambientais decorrentes da produção de bens e serviços e dos detritos gerados pelas regiões, a fim de constatar a existência de déficits ou potenciais reservas para serem gerenciadas.

Nesta perspectiva, a finalidade do referido método é estimar os impactos antrópicos no meio natural, mostrar a quantidade de área produtiva de terra e de água utilizada para fornecer produtos para abastecer a população de uma cidade, estado, região, ou país, sendo esta área de terra produtiva, ao mesmo tempo responsável por assimilar os resíduos gerados por essa mesma população (DIAS, 2002).

O instrumento de mensuração, já referido, é recomendado como ferramenta de trabalho por ser de fácil compreensão por todos aqueles que deles se utilizam. Essa ferramenta objetiva a mensuração da sustentabilidade ambiental, se preocupando com as agressões humanas sobre a natureza, a deteorização ecológica, além do que acaba ocasionando uma visão proativa para o homem sobre a importância e a necessidade de cuidar da água, do ar, das florestas, da flora e da fauna e se ter um limite de apropriação de produtividade da natureza (BELLEN, 2004).

De acordo com Wackernagel e Rees (1998), a essência do método da Pegada Ecológica está na contabilização dos fluxos de matéria e energia presentes em certo sistema. Este, por sua vez, é transformado em áreas de 
terra ou de água produtiva. Para os autores, a carga imposta por cada população varia em função de diversos aspectos não apenas econômicos, como os abordados na Pegada Ecológica, mas também de aspectos culturais e de produtividade ecológica.

A aplicação do método pode ser feita em uma pessoa ou em uma população e seus resultados irão depender de seus costumes, das características do dia-dia de cada indivíduo, seus padrões e de consumo. Estes fatores, por sua vez, influenciarão diretamente nos resultados provenientes da aplicação do método (WACKERNAGEL; REES, 1998).

\section{Procedimentos para cálculo do Ecological Footprint Method - EFM}

O método da pegada ecológica apresenta-se como uma ferramenta na avaliação da sustentabilidade. A aplicação do método sucede por meio de uma série de cálculos que, por sua vez, facilitam a observação da realidade estudada. Nesse contexto, Rees (1996) explica que o primeiro passo, no cálculo da pegada ecológica de uma dada população, é a estimativa da área apropriada para a produção dos principais itens de consumo; isto é feito dividindo-se a média anual de consumo de cada item pela produtividade média anual. $\mathrm{O}$ total per capita da pegada ecológica é o somatório dos itens de consumo considerados.

A metodologia de cálculo da pegada ecológica considera seis premissas principais (WACKERNAGEL et al., 2005):

1) Os montantes anuais de recursos consumidos e gastos gerados pela comunidade são monitorados por ações nacionais e internacionais;

2) A quantidade de recursos biológicos apropriados para uso humano é diretamente relacionada à quantidade de terra bioprodutiva necessária para regeneração e assimilação de resíduos;

3) Ponderando-se cada área em proporção à sua produtividade de biomassa utilizável (potencial anual de produção de biomassa utilizável), diferentes áreas podem ser expressas em termos de um hectare produtivo médio padrão;

4) A demanda total em hectares globais pode ser agregada, adicionandose todas as áreas provedoras de recursos e assimiladoras de resíduos requeridas para suportar a demanda;

5) A demanda humana agregada (pegada ecológica) e a capacidade natural (biocapacidade) podem ser diretamente comparadas uma com a outra;

6) A área de demanda pode exceder a área de fornecimento.

Sendo a pegada ecológica uma medida do impacto da população expressa em termos de área apropriada, sua finalidade é quantificar os fluxos de energia e massa de uma economia ou atividade específica, transformados em áreas correspondentes necessárias para suportar esses fluxos. Em outras palavras, o cálculo da pegada ecológica considera que para cada item de matéria ou energia consumida pela sociedade, existe certa área de terra, em um ou mais ecossistemas que é necessária para fornecer o fluxo de recursos e absorver os seus dejetos (WACKERNAGEL et al., 2005).

A pegada ecológica apresenta como unidade de medida o Global Hectar (gha). Esta unidade corresponde a um hectare de espaço biologicamente produtivo com "produtividade média mundial". A finalidade em se utilizar o Global Hectar no Ecological Footprint Method é proporcionar a 
comparação da pegada ecológica total entre países e regiões. Wackernagel et al. (1996 apud ANDRADE, 2006) mostram dois fatores de conversão almejando padronizar a produtividade por hectares em unidades de áreas globais, tais quais:

Equivalence Factor (Fator de Equivalência) - representa a produtividade média mundial de um determinado espaço ecológico bioprodutivo, dividido pela produtividade média mundial de todos os tipos de espaços ecológicos bioprodutivos;

Yeld Factor (Fator de Produção) descreve quanto um espaço ecológico bioprodutivo de um dado país é mais (ou menos) produtiva em referência a média mundial na mesma tipologia de espaço ecológico bioprodutivo. Há divergência no fator produção em se tratando de um país para outro, uma vez que cada país tem seus próprios fatores de produção para cada tipologia de espaço bioprodutivo. Na verdade, o fator de produção é a razão entre a área produtiva de um país, usada na produção de todos os itens de uma categoria estabelecida, mensurada através do uso de dados da produção mundial e a área que seria requerida casos os mesmos itens fossem produzidos com a média da produção mundial.

Estimando a utilização da terra é possível determinar a área total necessária para suportar o padrão de consumo humano. Nesse sentido Ribeiro et al. (2007) explica que devido à impossibilidade de estimar a demanda por área produtiva para provisão, manutenção e disposição de muitos bens de consumo, os cálculos ficam restritos às categorias mais importantes de bens e a alguns itens individuais. Dessa forma, o cálculo da área apropriada por certa população modifica de acordo com o número de itens escolhidos, assim como a disponibilidade de dados sobre o consumo.
Esses dados são padronizados para facilitar os estudos de casos e para estabelecer comparações entre regiões e países. Os procedimentos podem revelar pelo tamanho da "pegada ecológica" os efeitos das variações regionais dos padrões de consumo, produtividade e modelo de gestão, ajudando a identificar e eliminar erros e contradições no sistema.

Apesar das limitações, o método da Pegada Ecológica demonstra eficiência na sua aplicação e, principalmente, eficácia em seu resultado na medida em que contribui ativamente para disseminação do conhecimento acerca dos problemas ambientais. É esse conhecimento que proporciona a cada indivíduo a tomada de consciência, bem como a busca contínua pela sustentabilidade do planeta. Neste sentido, verifica-se a importância da avaliação da sustentabilidade de dado sistema, através do Ecological Footprint Method, como forma de atingir um equilíbrio na interação natureza e sociedade e, dessa forma, a alcançar a sustentabilidade (RIBEIRO et al., 2007).

\section{Materiais e Métodos}

A presente pesquisa trata de um estudo descritivo e exploratório efetivado através de um estudo de caso realizado na cidade de Campina Grande (PB), utilizando dados secundários, com formas de tratamento e análise de dados em conformidade com o estabelecido no método da pegada ecológica. Para a aplicação do método, foram escolhidos os principais itens de consumo, entre uma classificação de categorias que exerce uma grande pressão sobre as fontes produtoras de recursos naturais na região.

Os componentes de consumo selecionados para determinar o cálculo da 
Pegada Ecológica do município foram os seguintes: Energia elétrica, Água, Resíduos sólidos e Combustíveis. Tais itens foram escolhidos porque provocam intensos impactos negativos no meio ambiente, principalmente, os combustíveis (liberam gás carbônico e poluem o ar) e resíduos sólidos (produzem gás metano que contamina os solos e os lençóis freáticos).

As categorias de consumo selecionadas estão alinhadas às características prevalecentes no município por exercerem uma maior pressão ao sistema local, como também, contribuírem para o impacto negativo global do planeta.

Posteriormente, para uma análise mais consistente, procurou-se definir o saldo ecológico do município, considerando a diferença entre o Ecological Footprint Method das atividades realizadas pelo homem na região e a biocapacidade presente. $\mathrm{O}$ resultado final desta operação revela o total que o consumo humano está exigindo das fontes produtoras de recursos naturais.

A área objeto de estudo compreende a cidade de Campina Grande, situada no agreste paraibano e localizada a $120 \mathrm{~km}$ da capital do estado, João Pessoa. Campina Grande é o segundo município em população exercendo grande influência política e econômica sobre outros 57 municípios paraibanos. Atualmente, o município possui dentro do perímetro urbano algumas áreas verdes e além destas, três reservas ambientais, quais sejam: Parque Estadual do Poeta, Reserva do Louzeiro e a Reserva do Riacho das Piabas (O Parque Estadual do Poeta, com uma área de 419,51 hectares, e a Reserva do Loureiro, com uma área de 300 hectares).É importante ressaltar que as reservas do Louzeiro e do Riacho das Piabas estão passando por um intenso processo de devastação.

Tabela I. Discriminação dos tipos de consumo de energia elétrica no Município de Campina Grande - Ano Base (2007)

\begin{tabular}{lccccccc}
\hline \multicolumn{1}{c}{ Classe } & População & $\begin{array}{c}\text { Consumo } \\
\text { em kWh }\end{array}$ & $\begin{array}{c}\text { Consumo } \\
\text { em Gj }\end{array}$ & $\begin{array}{c}\text { EFM } \\
\text { (ha) } \\
\text { População }\end{array}$ & $\begin{array}{c}\text { EFM } \\
\text { (ha) } \\
\text { Per capita }\end{array}$ & $\begin{array}{c}\text { EFM } \\
\text { (gha) }\end{array}$ & $\begin{array}{c}\text { EFM } \\
\text { População }\end{array}$ \\
\hline \multicolumn{1}{c}{ Per capita } \\
\hline $\begin{array}{l}\text { Residencial } 381.422 \\
\text { Industrial }\end{array}$ & 381.422 & 126.668 .000 & $456.004,8$ & $4.560,048$ & 0,0119554 & $6.247,2658$ & 0,016379 \\
$\begin{array}{l}\text { Comercial } \\
\text { Rural }\end{array}$ & 381.422 & 77.485 .000 & 278.946 & 278.946 & 0,0073133 & $3.821,5602$ & 0,010019 \\
$\begin{array}{l}\text { Poder } \\
\text { público }\end{array}$ & 381.422 & 17.436 .000 & $62.769,6$ & 627,696 & 0,0016457 & 859,9435 & 0,002255 \\
$\begin{array}{l}\text { Iluminação } \\
\text { pública }\end{array}$ & 381.422 & 17.122 .000 & $61.639,2$ & 616,392 & 0,0016160 & 844,4570 & 0,002214 \\
$\begin{array}{l}\text { Serviço } \\
\text { público }\end{array}$ & 381.422 & 5.114 .000 & $18.410,4$ & 184,104 & 0,0004827 & 252,2225 & 0,000661 \\
$\begin{array}{l}\text { Consumo } \\
\text { próprio }\end{array}$ & 381.422 & 387.000 & $1.393,2$ & 13,932 & 0,0000365 & 19,0868 & 0,000050 \\
\hline \begin{tabular}{l} 
TOTAL \\
\hline
\end{tabular} & 381.422 & 559.319 .000 & 2.013 .548 & $20.135,4$ & 0,0527906 & $27.585,6131$ & 0,072323 \\
\hline
\end{tabular}

Fonte: IDEME, 2007 


\section{Resultados e Discussão}

Neste item encontram-se expostas as devidas análises realizadas para o atendimento do objetivo proposto. Dessa forma, a seguir encontram-se explicitadas uma série de tabelas com os resultados dos cálculos dos indicadores pertencentes às variáveis do método, quais sejam: energia elétrica, água, resíduos sólidos, combustível.

\section{Energia Elétrica}

$\mathrm{Na}$ tabela 1, estão apresentadas as classes de consumo de energia elétrica, a população, o total de consumo em $\mathrm{kWh}$ por classe, o consumo em $\mathrm{Gj}$ por classe, o EFM (Ecological Footprint Method) em hectares da população, o EFM em hectares per capita, o EFM em global hectare da população e o EFM em global hectare per capita.

Tomando como base o modelo de Parente (2007), os cálculos da variável consumo de energia elétrica foram realizados da seguinte maneira:

Obs.: Para facilitar a compreensão, as demonstrações numéricas estão sendo realizadas para classe residencial de consumo. Entretanto, estes cálculos devem ser feitos para todas as classes.

1) Busca de dados populacionais, de acordo com os dados fornecidos pelo IBGE (2008);

2) Consumo total de energia elétrica em kWh, referente ao ano de 2006 (IDEME);

3) Visando eficiência na realização dos cálculos referentes ao consumo de energia elétrica que é expresso em $\mathrm{kWh}$ e deve ser transformado em GJ utilizou-se um conversor denominado: Energy Unity Conversion Calculator. Entretanto, o cálculo pode ser feito através de uma regra de três simples. No exemplo abaixo, considere o consumo residencial de energia elétrica.

\begin{tabular}{c}
$1 \mathrm{kWh}-------------0,0036 \mathrm{Gj}$ \\
$126.668 .000 \mathrm{kWh}------\mathrm{X}=>$ \\
$\mathrm{X}=456.004,8$ Gigajoules \\
\hline
\end{tabular}

Para calcular o valor do Ecological Footprint Method em hectare da população EFM (ha) população, é necessário dividir o consumo em gigaloules por 100;

EFM (ha) população $=456.004,8 \div 100=4.560,048$

O Ecological Footprint Method em hectare per capita, item, foi calculado através da divisão do resultado do EFM (ha) população pelo total de habitantes;

EFM (ha) per capita $=4.560,048 \div 381.422=0,0119554$

O cálculo do Ecological Footprint Method em Global Hectare da população foi realizado multiplicando-se o $E F M$ (ha) da população, pelo Fator de Equivalência 1,37 atinente à biodiversidade global da terra de energia;

EFM (gha) população $=4.560,048 \times 1,37=$ $6.247,2658$

Calculou-se o Ecological Footprint Method em Global Hectare per capita dividindo-se o EFM (gha) população pelo total da população;

EFM (gha) per capita $=6.247,2658 \div 381.422=$
0,016379

Esses cálculos deverão ser realizados para todas as classes de consumo. Observe na tabela 1 , que uma vez realizados os procedimentos, ao seu final, na última linha denominada TOTAL é feito o somatório dos 
valores encontrados para as classes consideras. O somatório proveniete da coluna Ecological Footprint da população (ha) será utilizado para obter, a representação percentual do impacto que o consumo de energia elétrica no Ecological Footprint Total (ha). Sendo assim, a percentagem pode ser encontrada da seguinte forma:

Representação Percentual da variável no EFM Total (ha) = EFM (ha) da variável $\div$ Ecological Footprint Total

Ecological Footprint Total em hectares $=$ EFM (ha) de energia elétrica + EFM (ha) de água + EFM (ha) de resíduos sólidos + EFM (ha) de combustível

Em valores numéricos para o consumo de energia elétrica, obteve-se:

\begin{tabular}{|c|}
\hline Representação Percentual do Consumo \\
de energia elétrica da população no EFM \\
Total (ha) $=$ \\
$20.135,484 \div(20.135,484+487,974+$ \\
$100.860+309.362,557)=0,0467 \times 100=$ \\
$4,68 \%$ \\
\hline
\end{tabular}

É importante salientar que o percentual de impacto no Ecological Footprint Total (ha) somente será obtido quando todas as tabelas estiverem prontas, pois como ressaltado anteriormente, é necessário o Ecological Footprint Method (ha) da população de cada item (variável) de consumo.

Em se tratando do consumo de energia elétrica no município de Campina Grande, observou-se que o Ecological Footprint em hectare da população corresponde a 4,68\% do impacto negativo no ecossistema urbano, ou seja, de toda pressão imposta pela população no sistema urbano de Campina Grande, o consumo de energia elétrica expressou uma participação significativa de $4,68 \%$, valor que demonstra o elevado padrão de consumo por parte da população, sendo necessário substituir o estilo de consumo tradicional por formas de energia alternativas, por exemplo, a energia solar.

\section{Água}

A tabela 2 mostra as classes de energia, a população, o consumo de água $\mathrm{em}^{3}$, consumo de água em megalitros, o total de $\mathrm{CO}_{2}$ emitido em toneladas, Ecological Footprint Method da população em hectares, EFM per capita por hectare, EFM da população em global hectare e o EFM per capita por hectare.

Os cálculos desta variável foram realizados da seguinte forma:

Tabela 2. Discriminação dos tipos de consumo de água no Município de Campina Grande - Ano Base (2007)

\begin{tabular}{|c|c|c|c|c|c|c|c|c|}
\hline Classe & $\begin{array}{l}\text { Popu- } \\
\text { lação }\end{array}$ & $\begin{array}{c}\text { Consumo } \\
\mathrm{em} \mathrm{m}^{3}\end{array}$ & $\begin{array}{l}\text { Consumo } \\
\text { em (mgl) }\end{array}$ & $\begin{array}{c}\text { Total } \\
{\text { de } \mathrm{CO}_{2}}_{2} \\
\text { Emitido } \\
(\mathrm{t})\end{array}$ & $\begin{array}{l}\text { EFM/ } \\
\text { (ha) } \\
\text { Popu- } \\
\text { lação }\end{array}$ & $\begin{array}{c}\text { EFM/ } \\
\text { (ha) } \\
\text { Per capita }\end{array}$ & $\begin{array}{l}\text { EFM } \\
\text { Total } \\
\text { (gha) }\end{array}$ & $\begin{array}{c}\text { EFM } \\
\text { (gha) } \\
\text { Per capita } \\
\end{array}$ \\
\hline & A & $\mathrm{B}$ & $\mathrm{C}$ & $\mathrm{D}$ & $\mathrm{E}$ & $\bar{F}$ & G & $\mathrm{H}$ \\
\hline Residencial & 381.422 & 1.064 .150 & $1.064,15$ & 393,736 & 393,756 & 0010323 & 539,4176 & 0,0014142 \\
\hline ndu & & & & 28,798 & 28,798 & 0,000 & & 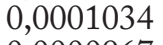 \\
\hline Comercial & 381 & 72.768 & 72,7 & 26,924 & 26,924 & 0,0000706 & 36,8861 & 0,0000967 \\
\hline $\begin{array}{l}\text { Poder } \\
\text { Público }\end{array}$ & 381.422 & 80.012 & 80,012 & 29,604 & 29,604 & 0,0000776 & 40,5581 & 0,0001063 \\
\hline $\begin{array}{l}\text { Misto } \\
\text { TOTAL }\end{array}$ & $\begin{array}{l}381.422 \\
381.422\end{array}$ & $\begin{array}{l}24.122 \\
1.318 .884,00\end{array}$ & $\begin{array}{l}24,122 \\
1.318,88\end{array}$ & $\begin{array}{l}8,925 \\
487,974\end{array}$ & $\begin{array}{l}8,925 \\
487,947\end{array}$ & $\begin{array}{l}0,0000234 \\
0,0012794\end{array}$ & $\begin{array}{l}\frac{12,2274}{668,5423} \\
\end{array}$ & $\begin{array}{l}0,0000321 \\
0,0017528\end{array}$ \\
\hline
\end{tabular}


Obs.: Para melhor compreensão, segue um exemplo elaborado com o valor referente ao consumo residencial de água. Todavia, todas as classes de consumo necessitam ser calculadas.

1) Levantamento da população, conforme os dados fornecidos pelo IBGE (2008);

2) Consumo total de água, referente ao ano de 2007 (Cagepa);

3) Conforme Chambers et. al apud Parente (2007), 01 (um) litro de água é igual a $0,001 \mathrm{~m}^{3}$ e 1 (um) mega litro corresponde a $1000 \mathrm{~m}^{3}$. Desta forma, o consumo de água em $\mathrm{m}^{3}$ pode ser transformado para $\mathrm{mgl}$ dividindo-se o valor em $\mathrm{m}^{3}$ por mil.

Consumo em mgl $=1.064 .150 \div 1.000=1.064,15$

4) Considerando que durante o processo de tratamento, encanação e distribuição de 01(um) mega litro de água as pessoas liberam $370 \mathrm{~kg}$ ou 0,37 toneladas de $\mathrm{CO}_{2}$ para a atmosfera, pode-se calcular o Total de $\mathrm{CO}_{2}$ Emitido ( $\mathrm{t}$ ), multiplicando o consumo em mgl por 0,37 (SILVA et al., 2010).

Total de $\mathrm{CO}_{2}$ emitido $(\mathrm{t})=1.064,15 \times 0,37=393,736$.

5) Segundo dados do IPCC, 01 (um) hectare absorve 01 (uma) tonelada de $\mathrm{CO}_{2}$. Assim, para calcular o EFM/(ha) população, divide-se o total de $\mathrm{CO}_{2}$ emitido toneladas (t) por 01.

$\mathrm{EFM} /$ (ha) população $=393,736 \div 01=393,736$

6) O cálculo do EFM/ (ha) per capita, dividindo-se o EFM/(ha) população pelo total de habitantes.

EFM $/$ (ha) per capita $=393,736 \div 381.422=0,0010323$
7) Para obter o valor referente EFM Total (gha), multiplica-se o EFM/(ha) população por 1,37 referente à produtividade da área da floresta.

EFM Total $($ gha $)=393,736 \times 1,37=539,4176$.

8) O EFM (gha) per capita foi calculado através da divisão do EFM Total (gha) pela população.

EFM (gha) percapita $=539,4176 \div 381.422=0,0014142$

Finalizados os cálculos para todas as classes de consumo de água, chegou-se ao total do EFM (ha) da população (487, 974), que evidencia o total de água que a população consumiu durante o ano. Esse total foi dividido pelo somatório dos totais correspondentes aos EFM (ha) da população das demais variáveis $(430.846,02)$ para encontrar o percentual de participação do consumo de água para degradação do planeta, ou seja, na pegada ecológica total.

Representação Percentual $=$ $487,974 \div 430.846,02=0,0011 \times 100=0,11 \%$

O Ecological Footprint em hectare da população referente ao consumo de água é representa $0,11 \%$ do impacto ambiental local. Esse percentual reflete uma conscientização por parte da população em se tratando do consumo de água. A população está consciente de que a água potável é um recurso escasso e que é preciso evitar desperdícios, almejando o equilíbrio do ecossistema e a sobrevivência das espécies.

\section{Geração de resíduos sólidos}

A tabela 3 apresenta classes, população, geração de resíduo total $(\mathrm{t})$, emissão de 


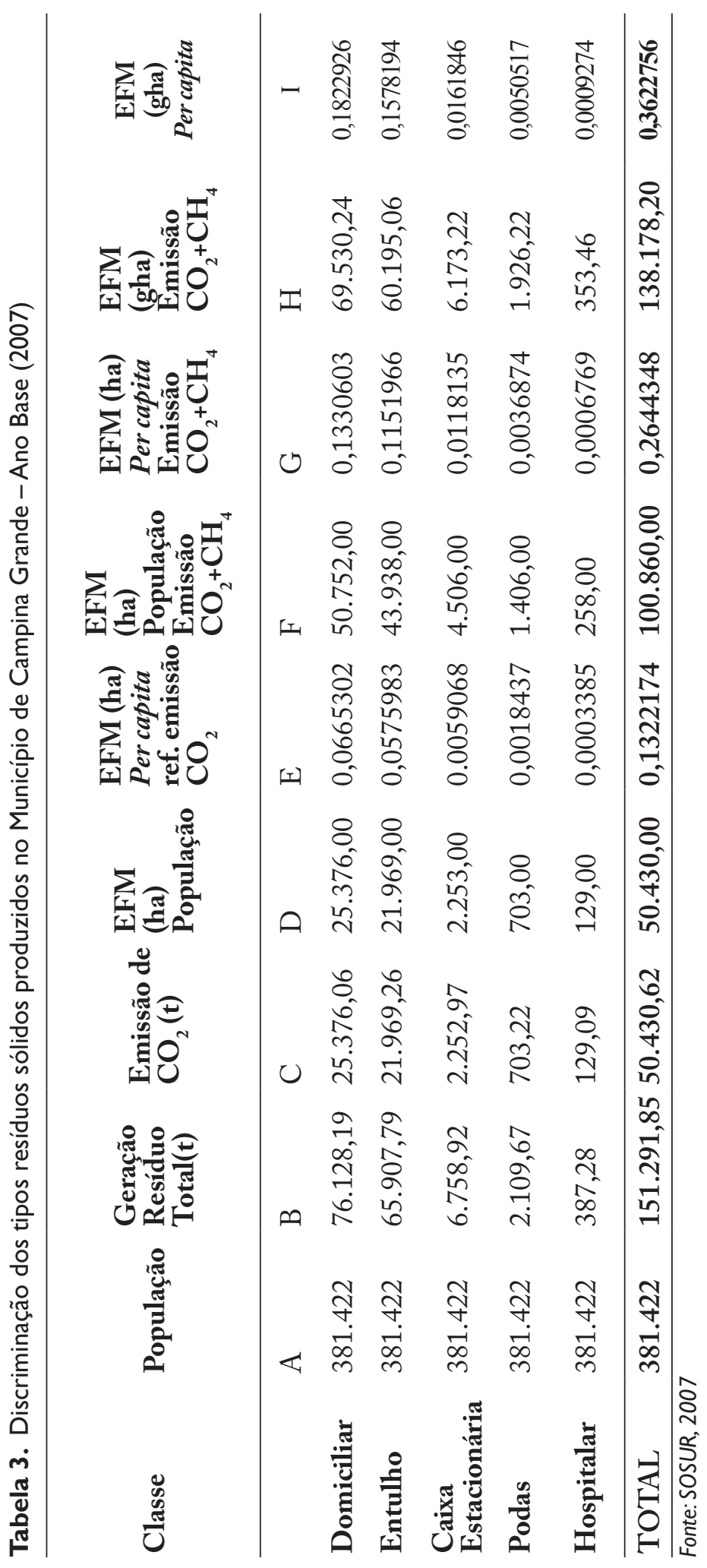

FEITOSA, M.J.S.; CÂNDIDO, G.A.; FIRMO, L.A. 
$\mathrm{CO}_{2}(\mathrm{t}), \mathrm{EFM}$ (ha) população, $\mathrm{EFM}$ (ha) per capita referente à emissão de $\mathrm{CO}_{2}, \mathrm{EFM}$ (ha) população emissão $\mathrm{CO}_{2}+\mathrm{CH}_{4}, \mathrm{EFM}$ (gha) Emissão $\mathrm{CO}_{2}+\mathrm{CH}_{4}$, EFM (gha) per capita.

Para obter os cálculos da variável resíduos sólidos, foram seguidos os seguintes procedimentos:

Obs.: Para melhor compreensão, segue um exemplo elaborado com o valor referente à geração de resíduo domiciliar.

1) Levantamento da população, conforme os dados fornecidos pelo IBGE (2008);

2) O volume de resíduos sólidos em toneladas, referentes ao ano de 2007 (SOSUR);

3) Conforme Andrade (2006), 0,00135 toneladas de resíduos produzem 0,00045 toneladas de $\mathrm{CO}_{2}$, assim a Emissão de $\mathrm{CO}_{2}$ em toneladas ( $\mathrm{t}$ ), pode ser calculada multiplicando-se a Geração de Resíduos Totais $(\mathrm{t})$ por 0,00045 . O resultado desta divisão deve ser dividido por 0,000135 . Através de uma regra de três simples:

$$
\begin{aligned}
& \text { 0,00135 (t)-----------0,00045 (t) de } \mathrm{CO}_{2} \\
& 76.128,19(\mathrm{t}) \text {-------- } \mathrm{X} \\
& \mathrm{X}=25.376,06 \text { toneladas de } \mathrm{CO}_{2}
\end{aligned}
$$

4) Segundo Silva, Correia e Cândido (2009) um hectare de terra absorve 01 (tonelada) de $\mathrm{CO}_{2}$. Assim, o Ecological Footprint Method em hectare é igual ao valor da emissão de $\mathrm{CO}_{2}$ em toneladas;

EFM (ha) da população $=25.376,00$

5) O Ecological Footprint Method em hectare per capita, foi calculado dividindose a Emissão de $\mathrm{CO}_{2}$ em toneladas ( $\mathrm{t}$ ) pela população da cidade;
EFM (ha) per capita ref. à emissão de $\mathrm{CO}_{2}=$ $25.376,06 \div 381.422=0,066530$

6) De acordo com Andrade (2006), a produção de $\mathrm{CO}_{2}$ está atrelada a geração de $\mathrm{CH}_{4}$. Sendo assim, no momento em que se produz um $\mathrm{kg}$ de $\mathrm{CO}_{2}$ gera-se também um $\mathrm{kg}$ de $\mathrm{CH}_{4}$. Dessa forma, para se obter o EFM (ha) População Emissão $\mathrm{CO}_{2}+\mathrm{CH}_{4}$, multiplica-se o EFM (ha) da população por 2;

EFM (ha) População Emissão $\mathrm{CO}_{2}+\mathrm{CH}_{4}$ $=25.376,00 \times 2=50.752,00$

7) Seguindo o critério anterior, o Ecological Footprint Method em hectare per capita, referente à emissão de $\mathrm{CO}_{2}+\mathrm{CH}_{4}$, foi calculado através da multiplicação do EFM (ha) per capita referente à emissão de $\mathrm{CO}_{2}$ por 02 ;

EFM (ha) per capita Emissão $\mathrm{CO}_{2}+\mathrm{CH}_{4}=$ $0,066530 \times 2=0,013306$

8) Para obter o EFM (gha) $\mathrm{CO}_{2}+\mathrm{CH}_{4}$, multiplica-se o EFM da população Emissão de $\mathrm{CO}_{2}+\mathrm{CH}_{4}$ por 1,37 referentes à Bioprodutividade Global da terra de energia;

EFM (gha) Emissão $\mathrm{CO}_{2}+\mathrm{CH}_{4}$ $=50.752,00 \times 1,37=69.530,24$

O EFM (gha) per capita, foi calculado através da divisão do resultado do EFM (gha) Emissão $\mathrm{CO}_{2}+\mathrm{CH}_{4}$ pela população;

EFM (gha) per capita $=$ $69.530,24 \div 381.422=0,1822922$

Ao término dos cálculos para todas as classes de resíduos, chegou-se ao total do EFM (ha) da população $(50.430,00)$, que evidencia o total de resíduos que a população gerou durante o ano. Esse total foi dividido pelo somatório dos totais correspondentes aos EFM (ha) da população das demais variáveis $(430.846,02)$ para obter o percentual de participação da geração de 
resíduos sólidos para degradação do planeta, ou seja, na pegada ecológica total;

Representação percentual $=$ $100.860 \div 430.846,02=0,2341 \times 100=$ $23,41 \%$

Dessa maneira, o Ecological Footprint por hectare da população correspondente à geração de resíduos sólidos é de $23,41 \%$ da composição da pegada ecológica do município. Esse percentual reflete o intenso volume de lixo produzido na cidade, em decorrência da aceleração do consumo por parte da população que nesse aspecto não se mostra preocupada com o desenvolvimento sustentável e a preservação do meio ambiente.

\section{Combustível}

A tabela 4 descreve o volume de combustível (álcool, diesel, gasolina e GNV), o total do volume de combustível, a emissão de $\mathrm{CO}_{2}$ emitido em toneladas, o EFM (Ecological Footprint Method) total por hectare, o EFM per capita por hectare, o EFM total por global hectare e o EFM per capita por global hectare.

Alguns critérios foram adotados para que fosse possível obter os resultados desejados. Tais critérios são:

Obs.: Para melhor compreensão, segue um exemplo elaborado com o valor referente ao consumo de álcool.

1) Levantamento da população, conforme os dados fornecidos pelo IBGE (2008);

2) Consumo total de combustível do município. As informações foram obtidas em relatórios da Agência Nacional do Petróleo (ANP), referente ao ano de 2006.

3) De acordo com Dias (2006),01 (um) litro de gasolina queimando libera 0,00263 (t) de $\mathrm{CO}_{2}$ (dióxido de carbono), 01 (um) litro de álcool emite 0,00252 (t), 01 (um) litro de diesel libera 0,00315 (t) e 01 (um) litro de Gás Natural Veicular (GNV) emite 0,00212 (t) de dióxido de carbono. Multiplicando a quantidade de litros demandada pelos valores expressos anteriormente, obtêm-se o total de toneladas de $\mathrm{CO}_{2}$ emitidos com o consumo de combustível no município;

$\left.\begin{array}{|l}\text { Consumo de álcool } \times 0,00252(\mathrm{t}) \\ \text { Consumo de diesel } \times 0,00315(\mathrm{t}) \\ \text { Consumo de gasolina } \times 0,00263(\mathrm{t}) \\ \text { Consumo de GNV } \times 0,00212(\mathrm{t})\end{array}\right\} \begin{gathered}\text { Emissão } \\ \text { de CO2 } \\ \text { em } \\ \text { toneladas }\end{gathered}$

Emissão de $\mathrm{CO}_{2}$ em toneladas

Para encontrar o Total de $\mathrm{CO}_{2}$ emitido em toneladas ( $\mathrm{t}$ ), multiplicou-se o consumo em litros por 0,00252 .

Total de $\mathrm{CO}$ emitido em $(\mathrm{t})=$ $4.608 .430 \times 0,00252=11.613,243$

4) De acordo com o relatório do IPCC (2007) (Intergovernmental Panel on Climate Change - Painel Intergovernamental de Mudança Climática) uma área de 1(um) hectare tem capacidade de absorver uma tonelada de $\mathrm{CO}_{2}$ emitida, portanto, o EFM (ha) da população é igual ao Total de $\mathrm{CO}_{2}$ emitido em $(t)$.

$\operatorname{EFM}($ ha) da população $=11.613,243$

5) Para calcular o EFM (ha) per capita, dividiu-se o EFM (ha) da população pelo total de habitantes da cidade.

EFM (ha) per capita $=$ $11.613,243 \div 381.422=0,0304$

6) Para obter o EFM Total (gha), multiplicou-se o EFM da população por 1,37, valor referente à produtividade global de terra. 
EFM Total $($ gha $)=11.613,243 \times 1,37=$ $15.910,143$

7) O cálculo do EFM (gha) per capita, foi efetivado pela da divisão do EFM Total (gha) pelo total de habitantes da cidade.

\begin{tabular}{c|}
\hline EFM (gha) per capita $=$ \\
$15.910,143 \div 381.422=0,0417$
\end{tabular}

Concluídos os cálculos para todos os tipos de combustível, pode-se obter o total do EFM (ha) da população $(309.362,557)$, que corrobora o total de combustível que a população consumiu anualmente. Este total foi dividido pelo somatório dos totais correspondentes aos 'EFM (ha) da população' das demais variáveis $(430.846,02)$ para obter o percentual de participação do combustível para degradação do planeta, ou seja, na pegada ecológica total.

$$
\begin{gathered}
\text { Representação percentual }= \\
309.362,557 \div 430.846,02=0,7180 \times 100= \\
71,80
\end{gathered}
$$

Os dados do Ecological Footprint por hectare da população, referentes ao volume de combustíveis consumidos em Campina Grande, representam o maior impacto no ecossistema global, 71,80\%. Esses dados refletem do aumento da frota dos veículos que utilizam combustíveis fósseis o que implica no aumento significativo da emissão de gases estufas na atmosfera, afetando negativamente o meio ambiente e comprometendo a sobrevivência das várias espécies existentes no planeta.

Uma vez efetivadas as análises a respeito de cada item de consumo, segue o cálculo do saldo ecológico que pode ser visualizado quando se faz a relação entre o total consumido pela população nas variáveis analisadas e o total de área verde que a cidade disponibiliza.

\section{Saldo/Déficit Ecológico}

De acordo com dados da Revista de Biologia e Ciências da Terra (2004), a cidade de Campina Grande tem uma média de 0,08 árvores para cada habitante. O município conta com áreas de preservação, como a Reserva do Louzeiro (300 hectares), Parque Estadual do Poeta (419,51 hectares), Reserva do Riacho das Piabas (240 hectares), conforme demonstra a tabela 5. Sendo que, o Parque Estadual do Poeta é a maior reserva de área urbana do Brasil.

Conforme a Lei 9.885 , que instituiu o Sistema Nacional de Unidades de Conservação da Natureza (SNUC), em 2000,

Tabela 4. Discriminação dos tipos combustível utilizados no Município de Campina Grande - Ano Base (2006)

\begin{tabular}{lccccccc}
\hline Itens & $\begin{array}{c}\text { Popu- } \\
\text { lação }\end{array}$ & $\begin{array}{c}\text { Consumo } \\
\text { em L }\end{array}$ & $\begin{array}{c}\text { Total } \\
\text { de CO2 } \\
\text { Emitido } \\
\text { (t) }\end{array}$ & $\begin{array}{c}\text { EFM/ } \\
\text { (ha) } \\
\text { População }\end{array}$ & $\begin{array}{c}\text { EFM/ } \\
\text { (ha) } \\
\text { Per } \\
\text { capita }\end{array}$ & $\begin{array}{c}\text { EFM } \\
\text { Total } \\
\text { (gha) }\end{array}$ & $\begin{array}{c}\text { EFM } \\
\text { (gha) } \\
\text { Per } \\
\text { capita }\end{array}$ \\
\hline Álcool & 381.422 & 4.608 .430 & $11.613,243$ & $11.613,243$ & 0,0304 & $15.910,143$ & 0,0417 \\
Diesel & 381.422 & 57.322 .419 & $180.565,619$ & $180.565,619$ & 0,4734 & $247.418,898$ & 0,6486 \\
Gasolina & 381.422 & 42.622 .313 & $112.096,683$ & $112.096,683$ & 0,2939 & $153.572,456$ & 0,4026 \\
GNV & 381.422 & 2.399 .534 & $5.087,012$ & $5.087,012$ & 0,0133 & $6.969,206$ & 0,0183 \\
\hline Total & 381.422 & 106.952 .696 & $309.362,557$ & $309.362,557$ & 0,8111 & $423.826,703$ & 1,1112 \\
\hline Fonte:ANP, 2006 & & & & & & &
\end{tabular}


Unidade de Conservação é o espaço territorial e seus recursos ambientais, incluindo as águas jurisdicionais, com características naturais relevantes. Tal espaço é legalmente instituído pelo Poder Público, com a finalidade de conservação e limites definidos, sob-regimes especiais de administração, ao qual se aplicam garantias adequadas de proteção.

Instituídas pelo poder público, as Unidades de Conservação podem se localizar em áreas públicas ou privadas. Podem ser federais, estaduais e municipais e, de acordo com a possibilidade de interferência humana no meio, são divididas em dois grandes grupos: unidades proteção integral e unidades de uso sustentável.
Ecological Footprint Method Totalé obtido pela somatória das áreas do Ecological Footprint por hectare da população do ecossistema, apropriadas por cada item de consumo. Destes valores totais são subtraídos os valores da biocapacidade (gha), representadas por áreas de conservação permanentes existente na cidade (Tabela 6).

A figura 1 apresenta os valores percentuais dos impactos causados pelas categorias de consumo no Ecological Footprint Total do Município de Campina Grande.

Portanto, as áreas de preservação na cidade, levando-se em conta as áreas silvestres protegidas, reservas biológicas e ecológicas

Tabela 5. Unidades de conservação existentes no Município de Campina Grande (PB)

\begin{tabular}{lccc}
\hline \multicolumn{4}{c}{ Unidades de conservação existentes em Campina Grande } \\
\hline \multicolumn{1}{c}{ Nome } & Área (ha) & Unidade de & Conservação \\
\hline Parque Estadual do Poeta & 419,51 & Estadual & Floresta Semi-Desidual \\
Reserva do Louzeiro & 300 & Municipal & Mata Nativa \\
Reserva do Riacho das Piabas & 240 & Municipal & Mata Nativa \\
\hline TOTAL & 959,51 & & \\
\hline
\end{tabular}

Fonte: IDEME, 2006.

Cada Unidade de Conservação recebe o manejo ambiental adequado para assegurar suas características naturais, ou seja: manter a diversidade natural, conservar os recursos genéticos e hídricos, favorecer a pesquisa científica, manejar os recursos florestais, promover a educação ambiental, o lazer, assegurar a qualidade ambiental e o crescimento econômico regional.

O saldo ecológico é obtido pela diferença entre o Ecological Footprint Method das atividades exercidas pelo homem em uma região e a biocapacidade presente. $\mathrm{O}$ resultado final desta operação revela o total da pressão imposta ao meio ambiente. Ou seja, com o uso limitado, perfazem um conjunto de 959,51 hectares, equivalentes a $1,55 \%$ da área total do município.

Os dados apresentados indicam que o Ecological Footprint Method Total da população do Município de Campina Grande é de 430.846,02 hectares e a área bioprodutiva é de 959,51 hectares, gerando um déficit Ecológico de 429.886,51 hectares. Esse valor evidencia o quanto as grandes cidades, sobretudo, os costumes de sua população prejudicam o ecossistema global. Em verdade, através da observação da realidade que permeia a cidade objeto de estudo, ratifica os resultados encontrados. 
Tabela 6. Resultado do Ecological Footprint Method Total do Município de Campina Grande

\begin{tabular}{cccc}
\hline Itens & População (hab.) & $\begin{array}{c}\text { Ecological Footprint } \\
\text { Total (ha) }\end{array}$ & $\begin{array}{c}\text { Representação } \\
\text { Percentual (\%) }\end{array}$ \\
\hline Energia Elétrica & 381.422 & $20.135,484$ & $4,68 \%$ \\
Água & 381.422 & 487,974 & $0,11 \%$ \\
Resíduos Sólidos & 381.422 & $100.860,00$ & $0,11 \%$ \\
Combustíveis & 381.422 & $309.362,557$ & $0,11 \%$ \\
\hline Total & 381.422 & $430.846,02$ & $100 \%$ \\
\hline
\end{tabular}

Fonte: resultado da pesquisa (2009)

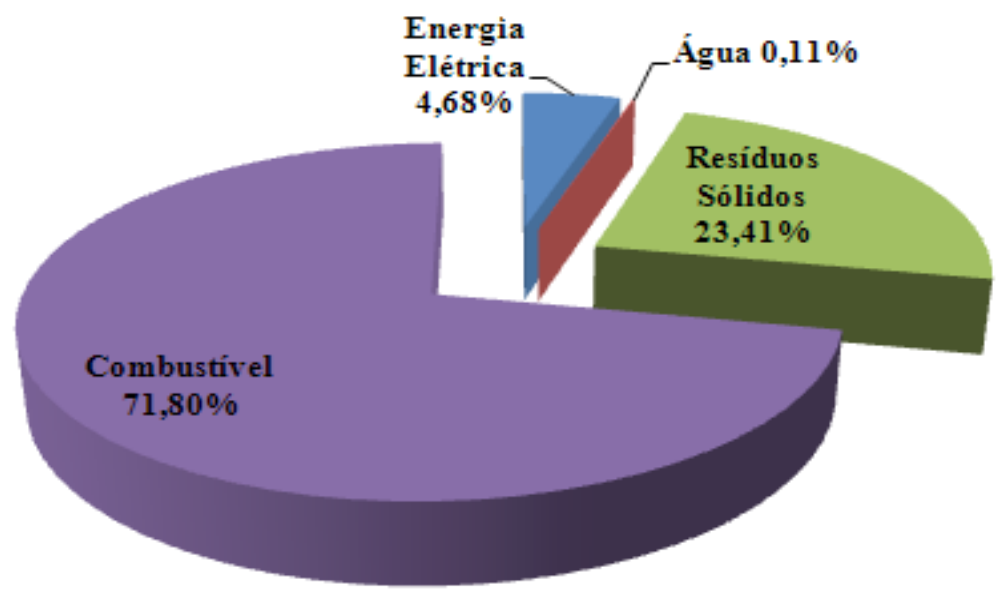

Figura I. Percentagem das categorias de consumo no Ecological Footprint Total de Campina Grande Fonte: resultado da pesquisa (2009)

\section{Considerações Finais}

Os resultados da aplicação do Ecological Footprint Method no município de Campina Grande corroboram a ideia de que as grandes cidades compostas por grande número de pessoas com hábitos insustentáveis deterioram o meio ambiente no qual elas estão inseridas.

A Pegada ecológica da cidade mostrou-se alta em virtude, sobretudo, da elevação do consumo de combustíveis $(71,80 \%)$ e da geração de resíduos sólidos $(23,41 \%)$. Tem-se observado que nos últimos anos a frota de automóveis cresceu bastante na cidade, devido ao aumento do poder aquisitivo da população. O crescimento da frota proporcionou a ascensão do consumo de combustível e, consequentemente, a emissão de gases estufa na terra. Por outro lado, observa-se na localidade alta produção de lixo. Este, por sua vez é despejado em um lixão municipal, poluindo o solo, os lençóis freáticos e o ar.

Outro fator que contribui sobremaneira para elevação da pegada ecológica da cidade objeto de estudo é a ausência de muitas unidades de conservação ou áreas florestais capazes de suprir as necessidades das pessoas e ao mesmo tempo absorver os resíduos produzidos. Em verdade, Campina Grande apresenta uma área verde ínfima em se comparando com o que seria necessário 
para suportar o padrão de consumo de sua população. As pesquisas realizadas mostram que a cidade apresenta apenas 959,51 hectares de área verde.

Para se ter uma ideia da dimensão do problema, os números obtidos no estudo mostram claramente que a população de Campina Grande requer uma área total de 430.846,02 hectares de áreas naturais para suprir as suas demandas por energia elétrica, água, resíduos sólidos e combustíveis e absorver os dejetos decorrentes da transformação desses elementos.

À medida que se contrapõe o déficit ecológico com o total de área verde que a localidade possui, conclui-se que seria necessário um total de área 449,03 (430.846,02: $959,51=449,03)$ vezes maior para suportar o metabolismo da cidade.

Neste sentido, corrobora-se a necessidade de ações urgentes para tomada de consciência por parte da sociedade, objetivando a redução e possível eliminação dos impactos ambientais na natureza. $\mathrm{O}$ descaso das pessoas acerca desta problemática implicará impossibilidade de sobrevivência humana no planeta.
Neste momento, percebe-se a importância do desenvolvimento contínuo de ferramentas capazes de auxiliar na gestão dos recursos naturais para um desenvolvimento sustentável. As ferramentas de mensuração da sustentabilidade, a exemplo do Ecological Footprint Method, dentro de suas limitações, refletem o contexto no qual o meio ambiente se encontra.

Assim, a pegada ecológica decorrente da avaliação dos itens/variáveis ratifica a insustentabilidade do Município de Campina Grande sob a perspectiva ambiental, bem como reflete os inadequados modos de consumo e o estilo de vida da população dessa cidade.

Para trabalhos vindouros seria recomendável, o acréscimo de indicadores que possam promover avanços no que se refere aos dados obtidos e ao método de estudo. Além disso, seria adequada a realização de outras aplicações da pegada ecológica em outras cidades do Estado da Paraíba, bem como, em outros estados nordestinos para que dessa forma seja possível analisar possíveis alterações de comportamento e comparar os dados com outras regiões do país.

\section{Referências}

ANDRADE, B. B. Turismo e sustentabilidade no Município de Florianópolis: uma aplicação do método de pegada ecológica. 2006. 152 f. Dissertação (Mestrado em Administração). UFSC - Universidade Federal de Santa Catarina-UFSC, FlorianópolisSC, 2006.

AGÊNCIA NACIONAL DO PETRÓLEO, GÁS NATURAL E BIOCOMBUSTÍVEIS - ANP. Disponível em: <http://www.anp.gov.br/doc/petroleo/abastecimento/Revenda/ volume_combustiveis_2006.pdf>. Acesso em: 25 mar. 2009.

BELLEN, H. M. V. Indicadores de Sustentabilidade: uma análise corporativa. Rio de Janeiro: FGV, 2007. 
Indicadores de Sustentabilidade: uma análise comparativa. 2002. 220f. Tese (Doutorado em Engenharia de Produção) - Universidade Federal de Santa Catarina, UFSC, Florianópolis, 2002.

. Desenvolvimento sustentável: uma descrição das principais ferramentas de avaliação. Ambiente \& Sociedade, v. 7, n. 1, p. 67-88, 2004.

COMPANHIA DE ÁGUA E ESGOTO DO ESTADO DA PARAÍBA - CAGEPA. Disponível em: <http://www.cagepa.pb.gov.br>. <pesquisa direta >

CIDIN, R. C. P. J; SILVA, R. S. Pegada Ecológica: instrumento de avaliação dos impactos antrópicos no meio natural. Estudos Geográficos, Rio Claro, v.2, n.1, p.43-52, 2004.

DIAS, G. F. Pegada Ecológica e Sustentabilidade Humana. São Paulo: Gaia, 2002. 257p.

DIAS, G. F. Pegada Ecológica e Sustentabilidade Humana. São Paulo: Gaia, 2006. 257p.

GONÇALVES, J. Propostas para um sistema de indicadores de desenvolvimento sustentável. Lisboa: Direção geral do ambiente, 2000.

INSTITUTO BRASILEIRO DE GEOGRAFIA E ESTATÍSTICA - IBGE. Indicadores de desenvolvimento sustentável: Brasil 2004. Coordenação de recursos naturais e estudos ambientais. Rio de Janeiro: IBGE, 2004.393p.

INSTITUTO BRASILEIRO DE GEOGRAFIA E ESTATÍSTICA - IBGE. Síntese dos indicadores sociais: uma análise das condições de vida da população brasileira. Rio de Janeiro: IBGE, 2008.

INSTITUTO BRASILEIRO DE GEOGRAFIA E ESTATÍSTICA - IBGE. Disponível em: <http://www.ibge.gov.br/cidadesat/topwindow.htm?1> Acesso em: 09 Mar. 2009.

INSTITUTO DE DESENVOLVIMENTO MUNICIPAL E ESTADUALDA PARAÍBA - IDEME. Anuário Estatístico do Estado da Paraíba. João Pessoa: IDEME, 2005.

INSTITUTO DE DESENVOLVIMENTO MUNICIPAL E ESTADUAL DA PARAÍBA - IDEME. Anuário Estatístico do Estado da Paraíba. João Pessoa: IDEME, 2006.

INSTITUTO DE DESENVOLVIMENTO MUNICIPAL E ESTADUAL DA PARAÍBA - IDEME. Anuário Estatístico do Estado da Paraíba. João Pessoa: IDEME, 2007. Disponível em: <http://www.ideme.pb.gov.br>. Acesso em: 24 maio 2009.

INTERGOVERNMENTAL PANEL ON CLIMATE CHANGE - IPCC. Fourth Assessment Report Working Group III. Mitigation of climate change, Bancoc, 2007.

McCOOL, S. F.; STANKEY, G.H. Indicators of Sustainability: Challenges and Opportunities at the Interface of science and Policy. Environmental Management, v. 33, n. 3, p. 234-305, Springer-Verlag. New York, 2004. 
MITCHELL, G. Problems and Fundamentals of sustainable Development Indicators. 1997. Disponível em: <www.lec.leeds.ac.uk/people/gordon.html>. Acesso em: 26 abr. 2009.

MORAES, $P$. R. As áreas tropicais unidas e as febres hemorrágicais virais: uma abordagem geográfica na área ambiental e na saúde. 2007.339f. Tese (doutorado em Geografia Física). Departamento de Geografia. Universidade de São Paulo, USP, 2007.

O'MEARA, M. Explorando uma Nova Visão para as Cidades. p.138-157. Estado do Mundo - 1999. Traduzido por Henry Mallet. Salvador: UMA, 2000.

PARENTE, A. Indicadores de Sustentabilidade Ambiental: um Estudo do Ecological Footprint Method do Município de Joinville - SC. 2007. 196f. Dissertação (Mestrado em Administração) - Departamento de Administração. Universidade do Vale do Itajaí, Biguaçu, 2007.

PENNA, C. G. O estado do planeta. Sociedade de consumo e degredação ambiental. Rio de Janeiro: Record, 1999. 256 p.

QUEIROZ, T. D. et al. Temas transversais e conteúdos \& conteúdos normais: Proposta Prática de Construção de Conhecimento Transversal: $1^{\circ}$ ciclo. São Paulo: Didática paulista, 2000.

REES, W. E. Revisiting Carrying Capacity: Area-Based Indicators of Sustainability. Population and environment, v. 17, n 3, p. 195-215, 1996.

REVISTA DE BIOLOGIA E CIENCIAS DA TERRA. Campina grande: Redalye, 2004. Arborização urbana na cidade de Campina Grande - PR: inventário e suas espécies. ISSN 1519-5228.

RIBEIRO,M.F.; PEIXOTO,J.A.A.; XAVIER, L. S. Estudo do Indicador de Sustentabilidade Pegada Ecológica: Uma Abordagem Teórico-Empírica. In: ENCONTRO NACIONAL DE ENGENHARIA DE PRODUÇÃO, 27., 2007. Anais... Foz do Iguaçu, 2007.

SILVA, A. M.; CORREIA, M. M.; CÂNDIDO, G. A ecological footprint method: avaliação de sustentabilidade do município de João Pessoa - PR. In: CÂNDIDO, G. A. (org.) Desenvolvimento sustentável e sistemas de indicadores. Campina Grande: UFCG, 2010.

SECRETARIA DE OBRAS E SERVIÇOS URBANOS - SOSUR. Disponível em: <http:// www.jaguarao.rs.gov.br>. <pesquisa direta>

SILVA, J. M.; SANTOS, J. R. Pegada ecológica: instrumento de avaliação dos impactos antrópicos no meio natural. Oecologia Brasiliensis, Rio de Janeiro, v. 11, n.4, p. 574 - 581, 2007. 
WACKERNAGEL,M.;MONFREDA,C.;MORAN,D.;WERMER,P.; GOLDFINGER, S.; DEUMLING, D.; MURRAY, M. National Footprint and Biocapacity Accounts 2005: The underlying calculation method. Global Footprint Network, Oakland, California, USA, 2005 .

WACKERNAGEL, M.; REES, W. Our Ecological Footprint: reducing human impact on the earth. Canada: New Society Publishers, 1996. 